Revue d'histoire de l'Amérique française

AlW REVUE D.HISTOIRE DE L'AMÉRIQUE FRANÇAISE

\title{
Jean de Lauson et Marie Gaudart : leur appartenance à une structure sociale
}

\section{Robert Le Blant}

Volume 23, numéro 1, juin 1969

URI : https://id.erudit.org/iderudit/302856ar

DOI : https://doi.org/10.7202/302856ar

Aller au sommaire du numéro

Éditeur(s)

Institut d'histoire de l'Amérique française

ISSN

0035-2357 (imprimé)

1492-1383 (numérique)

Découvrir la revue

Citer ce document

Le Blant, R. (1969). Jean de Lauson et Marie Gaudart : leur appartenance à une structure sociale. Revue d'histoire de l'Amérique française, 23(1), 110-121.

https://doi.org/10.7202/302856ar d'utilisation que vous pouvez consulter en ligne.

https://apropos.erudit.org/fr/usagers/politique-dutilisation/ 


\section{DOCUMENTS INÉDITS}

\section{JEAN DE LAUSON ET MARIE GAUDART : LEUR APPAR'TENANCE À UNE STRUCTURE SOCIALE}

Fils de François de Lauson, conseiller au Parlement de Paris et d'Isabelle Lotin, elle-même fille de Guillaume Lotin, maître ordinaire en la Chambre des Comptes de Paris, $\mathrm{Mr} \mathrm{Me}$ Jean de Lauson, conseiller au Parlement de Paris, seigneur de Lirec, épousa, suivant contrat du 13 avril $1614^{1}$, Marie Gaudart, née le 24 juillet $1598^{2}$. Elle était la fille de feu François Gaudart, conseiller au parlement, demeurant rue Vieille du Temple, paroisse Saint-Gervais, et de damoiselle Denise Canaye. Le fiancé fut assisté par messire François de Lauson, conseiller, aumônier ordinaire du roi, prieur du prieuré de Saint-Étienne d'Artz, en l'île de Ré, par Christophe de Lauson, écuyer, sieur de La Foucherie, par Me Joseph de Lauson, conseiller au parlement de Rennes, sieur de Feudet et de Jazeneuil, par Mr Me Guillaume Lotin, sieur de Charny, vicomte de Vaux, président des requêtes au Parlement, demeurant rue des Barres, paroisse Saint-Gervais, par $\mathrm{Mr} \mathrm{Me}$ Théodore Berzeau, sieur de Graves, conseiller au parlement, par messire Jean Bochart, sieur de Champigny, conseiller d'État, Mr Me Jean Bochart, sieur de Noroy, conseiller au parlement, Mr Me Jean Le Picart, sieur du Plessis, conseiller au parlement, par noble homme Me François Sublet, trésorier général des Finances à Rouen, par Mr Me Pierre Gayant, sieur de Varatre, conseiller au parlement, par Mr Me Pierre Berger, conseiller au parlement, par un certain Tronson, sans prénom ni qualification indiqués, par messire Christophe Hector de Marle, conseiller d'État, par Charles Surault, écuyer, sieur de Cherigny, gentilhomme ordinaire de la maison du roi.

1 XXVI: 32, aux Archives Nationales.

2 Acte du 24 mai 1622, minutes de Richer, LI : 136, idem. 
Ces témoins furent confondus sans précision comme parents, alliés et amis ainsi que ceux de la fiancée. Ces derniers furent, par contre, beaucoup plus nombreux, car ils comprirent noble homme Jean Rouillé, intitulé "sieur de" sans précision, Mr Me Jacques Godart, conseiller en la Cour de parlement, damoiselle Denise Gaudart, veuve de noble homme Me Papire Masson, avocat à la Cour, damoiselle Marguerite Gaudart, veuve de $\mathrm{Mr}$ Me Charles Tiraqueau, conseiller en la cour, damoiselle Louise de Compans, veuve de Mr Me Pierre Josse, conseiller du roi et maître ordinaire en sa Chambre des Comptes, damoiselle Marie Canaye, veuve de noble homme Me Pierre Dulac, vivant, avocat en ladite Cour, noble homme Me Charles Payot, conseiller, trésorier de la maison du roi, Mr Me Etienne Tournebus, conseiller au parlement, damoiselle Claude de Chaulnes, épouse de Philippe Canaye, écuyer, sieur de Poncourt, $\mathrm{Mr} \mathrm{Me}$ Jacques Chevalier, conseiller au parlement, $\mathrm{Mr}$ Me Nicolas Chevalier, conseiller au parlement, Me Simon Chevalier ${ }^{3}$, conseiller du roi, correcteur en la Chambre des Comptes, noble homme Me Jean Tiraqueau, avocat au parlement, Me Pierre Tiraqueau, sieur de Servan, conseiller du roi, trésorier de France et général de ses finances en Bourgogne, Mr Me Pierre Dulac, conseiller du roi et maître ordinaire en sa Chambre des Comptes, noble homme Me Antoine Feydeau, conseiller du roi et receveur général de ses finances en Poitou, $\mathrm{Me}$ Louis Dulac, avocat au parlement, $\mathrm{Me}^{4}$ de SainteMarthe, sieur de Champdoiseau, avocat au parlement, Me Pierre Manguin, avocat au parlement, messire François Le Roy, chevalier, sieur de La Grange, gentilhomme ordinaire de la chambre du roi, messire Anne de Créquy, chevalier, sieur baron de Riay, gentilhomme ordinaire de la chambre du roi, messire Jacques Danetz, sieur de Marly, conseiller d'État, président en la Chambre des Comptes, messire ${ }^{5}$ Olivier Le Fèvre, sieur d'Eaubonne, conseiller d'Etat, président en la Chambre des Comptes, $\mathrm{Mr} \mathrm{Me}^{6}$ Lefèvre, sieur d'Ormesson, conseiller du roi, maître des requêtes ordinaire de son hôtel, Mr Me Nicolas Lefèvre, conseiller du roi en sa cour de parlement, commissaire ès requêtes du Palais,

3 Le nom est abrégé dans le manuscrit.

4-6 Espace blanc dans le manuscrit. 
noble homme Me Nicolas de Villoutreys, conseiller du roi, trésorier de l'extraordinaire des guerres, $\mathrm{Mr}$ Me Jubert, sieur du Thil, conseiller du roi, maître des requêtes ordinaires de son hôtel $^{7}$ de Lommenie, sieur ${ }^{8}$, noble homme, Me Mathieu Prévost, grenetier du sel à Paris, $\mathrm{Me}^{9}$ Picot, conseiller du roi, trésorier de France et général de ses finances à Amiens, $\mathrm{Mr}^{10}$ Payen, conseiller, notaire, secrétaire du roi, maison et couronne de France, Me Abel Bourneau, conseiller du roi, substitut de $\mathrm{Mr}$ le Procureur général.

Le contrat fut donc passé en présence de quatorze témoins pour Lauson et de trente-deux autres pour sa fiancée. Celle-ci apporta 60,000 livres, somme dont une moitié lui était acquise par la succession de son père; l'autre résultant d'un avancement d'hoirie sur celle de sa mère qui conservait, par contre, sa vie durant, l'usufruit des biens de son mari. Seize mille livres seulement entrèrent en communauté, le reste devant être remployé en propre de la fiancée que Lauson gratifia comme douaire préfixe d'une rente de 1,200 livres, réduite à 1,000 en cas de survenance d'enfant. Les fiancés s'assurèrent pour chacun d'eux un privilège portant sur 4,000 livres en cas de partage de communauté, lui pour ses livres, armes et chevaux, elle, pour ses vêtements, bagues et joyaux. Marie Gaudart pourrait, en refusant la communauté, reprendre tout ce qu'elle aurait apporté, même par succession.

L'importance nominale de la dot, payée en espèces, le surlendemain, révèle l'existence d'une fortune considérable au profit de la famille Gaudart, surtout si la fiancée avait des frères et sœurs, ce que nous rechercherons plus loin. Seuls le privilège de 4,000 livres et l'importance du douaire représentant un capital d'environ 20,000 livres nous renseignent sur le pouvoir d'achat de Jean de Lauson à cette époque. Les clauses du contrat sont, au demeurant, normales, suivant les usages parisiens du temps. L'indication des parents nous permet de définir le contrat de mariage de Jean de Lauson comme étant celui d'un conseiller au parlement, fils d'un conseiller au parlement épousant la fille d'un conseiller au parlement, mais l'absence d'autre indication

7-10 Ibid. 
portant sur les parents et alliés va nous obliger, pour situer le milieu social des fiancés, à rechercher quels furent les éléments constitutifs de la famille.

Les omissions du rédacteur de l'acte furent malheureusement d'ordres divers, portant sur les identités comme sur les qualifications. Un témoin de la fiancé figura sans prénom ni qualification. Un de ceux du fiancé perdit son prénom, deux autres furent qualifiés "sieurs de" sans précision de nom de lieu. L'abréviation d'un nom de famille a pu faire l'objet d'un rétablissement selon d'autres passages de l'acte, le coefficient des omissions notariales reste donc fixé à quatre quarante sixièmes, soit environ $9 \%$. Ce taux négligeable ne doit pas empêcher la recherche, d'après l'acte lui-même, d'éclaircissements sur la situation sociale des fiancés par le moyen de deux statistiques: la première portant sur les professions des témoins; la seconde sur leurs qualifications. D'après les programmes généraux des derniers congrès des sociétés savantes françaises pour l'histoire, depuis 1610, les professions constituent des structures sociales. Il semblerait que l'application de ce principe doive être entendue au sens large et que l'absence de profession doive constituer aussi une structure sociale, bien que certains puissent la trouver critiquable.

La situation des femmes va créer ici une difficulté. Bien qu'aux termes du contrat, elles soient considérées comme n'exerçant aucune profession, nous n'avons pas crû devoir rapprocher de celle des magistrats une structure par omission de labeur et nous avons tenu compte de la profession du mari, toujours indiquée, même lorsqu'il s'agit d'une veuve. Cette solution trouve un appui, si on estime qu'au dix-septième siècle, la structure sociale constituée par les magistrats comportait, suivant les conceptions du temps, une absence de travail professionnel pour leurs épouses, les soins domestiques n'étant pas alors considérés comme constituant une profession indépendante telle que celle de ménagère. Elle prête cependant à la critique, puisqu'elle semble faire intervenir comme témoins des personnages décédés. D'autre part, l'absence, pour certaines femmes, d'une profession autre que celle de ménagère ou de maîtresse de maison, doit, 
évidemment, selon certaines méthodes actuelles, être recherchée par statistique, mais selon nous, au cours d'études sur l'ensemble des structures sociales et non au sein d'une structure dans laquelle elle était de principe.

C'est dans ces conditions de rédaction que Jean de Lauson fut assisté par six conseillers au parlement, un président au parlement et deux conseillers d'État, par un trésorier général des Finances, un gentilhomme ordinaire de la maison du roi et deux derniers témoins dont la profession n'est pas indiquée, soit, sur quatorze témoins, par douze titulaires d'offices représentant une majorité d'environ $86 \%$ et par neuf robins représentant une majorité d'environ $65 \%$. Pour la fiancée, les témoins appartenant à la robe dominèrent également avec sept conseillers au parlement, six avocats au parlement, un substitut du procureur général, deux maîtres en la Chambre des Comptes, un correcteur en la même Chambre, deux maîtres des requêtes, deux conseillers d'État, présidents en Chambre des Comptes. Ils furent complétés par cinq représentants du personnel des Finances, soit trois receveurs généraux des Finances dans une province, un trésorier de la maison du roi et un trésorier de l'extraordinaire des guerres, par un grenetier du sel, par un secrétaire du roi, trois gentilshommes ordinaires de la Chambre du roi et trois témoins dont la profession n'est pas indiquée. Les titulaires d'offices, au nombre de vingt-neuf sur trente-deux témoins présentèrent une majorité d'environ $91 \%$; les robins, au nombre de vingt-et-un, une majorité de $65 \%$, comme pour Jean de Lauson.

En ce qui concerne les cinq témoins figurant sans indication de profession, à raison de deux pour Lauson et trois pour la fiancée, il s'agit de Christophe de Lauson, écuyer, sieur de La Fourcherie, d'un Tronson, non autrement identifié, de noble homme Jean Rouillé, sieur d'un lieu non indiqué, de Philippe Canaye, écuyer, sieur de Poncourt, et d'un Loménie porté sans autre précision. On ne peut faire autrement, pour une statistique fondée sur le contrat de mariage, que de l'éliminer avec Tronson pour le classer hors statistique parmi les omissions imputables à la rédaction notariale. 
Nous restons en présence de trois personnages dont deux qualifiés écuyers et un noble homme, mais tous trois qualifiés "sieurs de". Il apparaît donc qu'une statistique des qualifications est susceptible de compléter celle des professions. Elle peut, en tout cas, renseigner sur la considération sociale dont bénéficiaient, à l'époque, les représentants des professions et faciliter la détermination d'une séparation entre nobles et roturiers indiquée par certains auteurs, avec d'autant plus d'opportunité que ces qualifications vont porter sur un certain nombre de tenanciers d'offices de judicature considérés par d'autres comme constituant un quatrième ordre, s'élevant au-dessus du Tiers État, pour atteindre le niveau inférieur de la Noblesse ${ }^{11}$.

En ce qui concerne Jean de Lauson, intitulé " $\mathrm{Mr} \mathrm{Me}$ " et "seigneur de", il fut assisté par un "Me", un "noble homme", un "Mr Me", un "Me sieur de", cinq "Mr Me sieur de", deux "écuyers, sieurs de", deux "messires", un "messire, sieur de" et un "Mr Me sieur de, vicomte de". La majorité appartient aux "Mr Me sieur de" dans une proportion de cinq quatorzièmes, soit environ $28 \%$. La qualification la plus employée sans tenir compte des adjonctions est celle de "Maître" avec neuf quatorzièmes, soit environ $65 \%$.

Pour Marie Gaudart, nous trouvons un "Monsieur", un "sieur", cinq "Maîtres", trois "nobles hommes", un "sieur de", deux "Monsieur-Maître sieur de", un "Maître sieur de", quatre "noble homme-maître", neuf "Monsieur-Maître", un "noble homme sieur de", un "écuyer sieur de", un "Messire sieur de", un "Messire chevalier sieur de", un "Messire chevalier baron de". Les "Monsieur-Maître" sont en majorité de neuf trente-troisièmes, soit environ $36 \%$. La qualification la plus employée, sans tenir compte des adjonctions est encore "Maître", avec treize trente-troisièmes, soit environ $54 \%$. On remarque que les qualifications comprennent d'une façon très nette celles qui précèdent le nom et celles qui le suivent.

Une étude tenant compte de cette distinction peut être tentée. Les qualifications précédant le nom patronymique pou-

11 Cf. Roland Mousnier, La Vénalité des offices sous Henri IV et Louis $X I I I$ (sans date, vers 1946), 59 et 60 . 
vant être simples ou doubles, les premières sont les moins nombreuses comprenant, pour Jean de Lauson, un maître et trois messires. Le maître est un conseiller au parlement, les trois messires sont un aumônier ordinaire du roi et deux conseillers d'État. Pour Marie Gaudart, un monsieur est un secrétaire du roi; sept maîtres sont trois avocats, deux trésoriers généraux, un trésorier de l'extraordinaire des guerres et un substitut du Procureur général; un seul noble homme est sans profession indiquée; quatre messires sont deux gentilshommes ordinaires de la Chambre du roi et deux conseillers d'État.

Les qualifications doubles sont monsieur-maître et noble homme-maître. Pour Lauson, les sept messieurs-maîtres sont un président et six conseillers au parlement. Un noble-hommemaître est un trésorier général des Finances à Rouen. Pour Marie Gaudart, dix messieurs-maîtres sont six conseillers au parlement, deux maîtres des requêtes et deux maîtres en la Chambre des Comptes; cinq nobles hommes-maîtres sont deux avocats, un trésorier de la maison du roi, un trésorier général des Finances et un grenetier du sel. Trois témoins sont sans qualification précédant le nom patronymique. Une hiérarchie dans l'ordre de ces qualifications préliminaires semble établie, pour l'ensemble des témoins, dans l'ordre suivant: la qualification "Monsieur", peu usitée, concerne un secrétaire du roi qu'on croirait plutôt trouver "noble homme"; la qualification "maître" concerne trois avocats, un conseiller au parlement, deux trésoriers généraux, un trésorier de l'extraordinaire des guerres et un substitut du procureur général; celle de "noble homme" peu usitée s'applique seulement à deux personnages dont la profession n'est pas indiquée; la combinaison "noble homme-maître" sert à deux avocats, deux trésoriers généraux des finances, un trésorier de la maison du roi et un grenetier du sel; le bloc de dix-huit "messieurs-maîtres" est constitué par un président au parlement, douze conseillers au parlement, deux maîtres des requêtes et deux maîtres en la Chambre des Comptes. Les sept "messires" sont un aumônier du roi, deux gentilshommes de la Chambre du roi et quatre conseillers d'Etat. 
Les qualifications placées à la suite du nom patronymique comprennent "sieur de", "sieur de, écuyer", "chevalier, sieur de", "chevalier, sieur baron de", "chevalier, sieur de, vicomte de". Les "sieurs de" qualifient six conseillers au parlement, un avocat, deux conseillers d'État, trois maîtres des requêtes, soit onze témoins. On aurait tendance à penser qu'il s'agit d'une qualification appliquée indépendamment de leurs professions aux possesseurs de terres, mais il est matériellement évident qu'elle porte sur les degrés supérieurs de la structure sociale hiérarchisée. Les "sieurs de, écuyers" sont Christophe de Lauson, écuyer, sieur de La Foucherie, Charles Surault, écuyer, sieur de Cherigny, gentilhomme ordinaire de la Chambre du roi, Philippe Canaye, écuyer, sieur de Poncourt. Etant donné qu'on ne trouve pas d'écuyer qui ne soit aussi qualifié "sieur de", il faut bien envisager l'existence d'un lien entre ces deux qualifications. Le "chevalier, sieur de" est François Le Roy, chevalier, sieur de La Grange, le "chevalier, sieur baron de" est Anne de Créquy, chevalier, sieur baron de Rizy, le "sieur de, vicomte de" est Guillaume Lotin, sieur de Charny, vicomte de Vaux. Les deux premiers sont gentilshommes de la Chambre du roi. Guillaume Lotin est président au parlement de Paris. Le lien entre les qualités de "sieur de" et d' "écuyer" paraît bien constituer une prétention vers la noblesse, renforcée lorsque le lien est entre la qualification de "chevalier" et celle de "sieur de", plus encore lorsque le lien est entre "chevalier" et "sieur baron de" plus encore lorsque le lien est entre "sieur de" et "vicomte de".

Les historiens opposent habituellement à l'anoblissement obtenu par l'acquisition d'une terre, l'existence du droit de francfief imposé aux roturiers acquéreurs de terres nobles, mais les notaires du début du XVIIe siècle semblent avoir eu l'esprit plus libéral en appliquant des qualifications même titrées aux possesseurs de terres. Le fiancé ayant seul été qualifié "seigneur de", la libéralité paraît, ici encore, avoir été courtoise vis-à-vis des parties ${ }^{12}$. La profession de gentilhomme ordinaire de la Chambre du roi peut aussi avoir été prise en considération, mais

12 Actes du 87e congrès des Sociétés Savantes (Paris, 1963), 672-673. 
elle ne fut pas déterminante, car Charles Surault fut qualifié "écuyer, sieur de", François Le Roy, "chevalier, sieur de". Les contrats de mariage du XVIIe siècle sont précieux pour l'identification des personnes et l'orthographe des noms à condition de se reporter aux signatures, la rédaction des actes étant souvent particulièrement négligée à ce dernier point de vue. Les Lauson, habituellement dénommés Lauzon, signèrent Lauson et les Gaudart, Gaudart, bien qu'on rencontre souvent l'orthographe Godart. Il ne paraît pas, cependant, pouvoir être contesté que les indications fournies par les contrats de mariage puissent être complétées à l'aide d'autres documents, bien que le résultat de telles recherches particulièrement utiles pour la reconstitution des familles, semble devoir rester imparfait, faute d'indications suffisantes portées au contrat de mariage, les noms patronymiques pouvant seulement constituer des présomptions servant de points de départ. Nous trouvons trois Lauson, François II et Christophe, frère du fiancé ${ }^{13}$, Joseph que nous n'avons pu rattacher. L'absence de tous autres parents ou alliés du côté paternel peut s'expliquer en partie par l'origine poitevine de la famille Lauson dont deux représentants seulement vinrent s'installer à Paris au XVIe siècle.

La très importante famille Lotin ne paraîtrait représentée que par Guillaume IV Lotin ${ }^{14}$, oncle du fiancé. Mais il faut y ajouter Théodore Berzeau, son cousin germain par alliance, comme époux de Jeanne Lotin, Christophe Hector de Marle, allié par le mariage de son frère Jacques ${ }^{15}$, avec Bonne Lotin, tante du fiancé. Jean IV Bochart, sieur de Champigny et Jean V, son fils, intitulé sieur de Noroy, mais qui lui succéda comme sieur de Champigny et fut le grand-père de l'intendant du Canada étaient alliés à Jean de Lauson ${ }^{16}$ par le mariage de Jeanne Bochart, leur tante et grand-tante, avec Guillaume III Lotin,

13 Inventaire des biens de François Ier de Lauson, du 17 novembre 1611, XXVI: 42.

14 Jean Sterlin, Chroniques d'histoire locale. Bulletin paroissial de Vaires-sur-Marne, 17 février 1956 et Dossiers Bleus, art. Lotin, à la Bibliothèque Nationale.

${ }_{15} \mathrm{La}$ Chesnaye-Desbois, Dictionnaire de la Noblesse, art. Hector et Pièces originales 1501, art. Hector.

16 La Chesnaye-Desbois, op. cit., art. Bochart. 
son grand-père. Les Bochart étaient cause d'autres alliances, avec Pierre Gayant, par suite du mariage de Françoise Gayant, et d'Antoine Bochart, arrière-grand-père de madame de Lauson; avec François Sublet, par le mariage de Jean Sublet et de Madeleine Bochart, nièce de Jeanne Bochart, elle-même grand-mère de Jean de Lauson; vraisemblablement avec le représentant non identifié de la famille Tronson, par le mariage de Jeanne Tronson avec Jean III Bochart, père de madame Lotin. Pour les témoins du fiancé, il n'en reste que quatre: Joseph de Lauson, Jean Le Picart, sieur du Plessis, Pierre Berger et Charles Surault dont l'intégration dans la famille n'est pas établie, soit une proportion de 4/14èmes et d'environ $28 \%$, pour les étrangers, $72 \%$, pour la famille. Pour la fiancée, l'importance de sa famille paternelle ne ressort pas à priori, du frère et des deux tantes énoncés au contrat, Jacques, Denise Gaudart, veuve de Papire, alias Papirius ${ }^{17}$ Masson, Marguerite Gaudart, veuve de Charles Tiraqueau. Ce dernier mariage fut vraisemblablement la cause d'une alliance avec Pierre et Jean Tiraqueau. Les mariages de deux autres tantes avaient entraîné des alliances avec François Le Roy, par l'union de Françoise Gaudart et de Jacques Le Roy; avec Jacques, Nicolas II et Simon Chevalier, par l'union de Madeleine Gaudart avec Nicolas Ier Chevalier. Il faut ajouter le mariage de Madeleine Ière Gaudart, grand-tante de la fiancée, avec Jean Lefèvre, cause d'alliance avec trois petits-fils de ce dernier, Olivier, André, sieur d'Ormesson, maître des requêtes, Nicolas, conseiller au parlement ${ }^{18}$. Enfin, Louise de Compans, veuve de Pierre Josse, était alliée par le mariage de Jean Gaudart, grand-père de la fiancée, avec Catherine Josse.

La famille maternelle de Marie Gaudart ne comprit que deux représentants dénommés Canaye: Philippe, son oncle et Marie, sa cousine. L'alliance avec Étienne II Tournebus provient du mariage du père de ce dernier, Etienne Ier Tournebus, avec Suzanne Canaye, fille de Philippe et cousine germaine de la fiancée. Le mariage de Marie Canaye entraîne une alliance plus lointaine avec Pierre II Dulac, neveu de son mari, Pierre Ier

17 Cabinet d'Hozier 156, art. Gaudart.

18 Dossiers Bleus 267, art. Lefèvre, à la Bibliothèque Nationale. 
Dulac, ainsi qu'avec Louis Dulac et Pierre Payot, par suite du mariage de ce dernier avec Marguerite Canaye, tante de Pierre II Dulac et probablement parente lointaine de Marie Gaudart. Il faut ajouter l'alliance d'Abel Bourneau causée par le mariage d'Elisabeth Bourneau avec Jean Canaye, oncle de Marie Gaudart, celle de Jean Rouillé par suite du mariage de Denise Rouillé, sa tante, avec Pierre Canaye, grand-père de la fiancée, celle de Jacques II Danès, par suite du mariage de Jacques Ier Danès avec Marie Rouillé, sœur de Denise ${ }^{19}$. Dix témoins de la fiancée qui n'ont pas été rattachés par des liens familiaux subsistent donc: Feydeau, de Sainte-Marthe, Manguin, de Créquy, de Villoutreys, Jubert, de Lomenie, Prévost, Picot et Payen, soit un pourcentage de vingt-trois trente-troisièmes et de $69 \%$ en faveur de la reconstitution de la famille, bénéficiant ainsi d'un pourcentage presque constant pour l'ensemble des témoins d'environ $70 \%$. Il faut préciser que, tant pour le fiancé que pour la fiancée, la famille est très loin d'être exactement indiquée, puisqu'un frère et une sœur de Jean de Lauson, trois frères de Marie Gaudart sont passés sous silence par le contrat, comme les représentants des importantes familles alliées, de Malebranche, Huault de Montmagny et Parent de Villemenon.

\section{Conclusion}

Le contrat de mariage de Jean de Lauson et de Marie Gaudart indique qu'ils appartenaient à une structure sociale homogène, constituée au début du XVIIe siècle à Paris par des titulaires d'offices importants, comprenant au sommet des magistrats quelquefois improprement appelés par des écrivains modernes, "titulaires d'offices de judicature". Les représentants de cette structure sociale, pourvus d'un pouvoir d'achat considérable étaient qualifiés le plus souvent "Monsieur-Maître" et tendaient vers la noblesse réelle.

Les difficultés rencontrées pour suppléer aux omissions de l'acte et rétablir la structure de la famille établissent à contrario l'importance des contrats de mariage en cette matière. Elles

19 Nouveau d'Hozier 156, idem et La Chesnaye-Desbois, Dictionnaire de la Noblesse, art. Canaye. 
peuvent cependant suggérer une possibilité de considérer comme assez aléatoires les résultats pouvant être obtenus par l'application des méthodes modernes de recherches par statistiques, portant sur une catégorie d'actes déterminée. L'importance des dots ne peut être exactement appréciée qu'avec une connaissance certaine du nombre des enfants pourvus. Les rédactions de généalogies, aussi complètes que possible, comportant en outre des précisions utiles pour les historiens sociaux, ne nous paraissent pas encore devoir être abandonnées.

Paris, France

ROBERT LE BLANT

\section{LIVRES REÇUS :}

Marc Rondeau, La promotion de la femme dans la pensée de l'Eglise contemporaine. Fides, 245 est, boul. Dorchester, Montréal. 291 p. \$5.00.

Le Chrétien à la barre des témoins - Colloque des responsables sociaux du Canada français, organisé par le Département d'Action sociale de la C.C.C. et tenu à Montréal du 21 au 23 novembre 1968. Fides, Montréal 129. 180 p. $\$ 3.00$.

Solange Chaput-Rolland, Regards '68 - Une ou deux Sociétés justes? Le Cercle du Livre de France, 1969, 215 p.

B. A. Keys, D. S. Rothwell, F. G. Thompson, Relevé des projets d'investissement à moyen terme, 1968. Le présent document a été préparé par des membres du personnel du Conseil économique du Canada.

Réginald Hamel, Cahier bibliographique des lettres québécoises, 2, avril, mai, juin 1968. Centre de documentation des lettres canadiennesfrançaises, Université de Montréal. $\$ 6.00$ le no; abonn. 4 nos: $\$ 20.00$.

Louis Baudouin, La recherche au Canada français. Textes des communications présentées au Colloque de la Section des lettres et des humanités, lors de la réunion annuelle de la Société royale du Canada, 1968. Les Presses de l'Université de Montréal, c.p. 6128, Montréal 101. 